\title{
Metasecretome-selective phage display approach for mining the functional potential of a rumen microbial community
}

\author{
Milica Ciric ${ }^{1,2}$, Christina D Moon ${ }^{1}$, Sinead C Leahy ${ }^{1}$, Christopher J Creevey ${ }^{3}$, Eric Altermann ${ }^{1}$, Graeme T Attwood ${ }^{1}$,
} Jasna Rakonjac ${ }^{2 *}$ and Dragana Gagic ${ }^{1 *}$

\begin{abstract}
Background: In silico, secretome proteins can be predicted from completely sequenced genomes using various available algorithms that identify membrane-targeting sequences. For metasecretome (collection of surface, secreted and transmembrane proteins from environmental microbial communities) this approach is impractical, considering that the metasecretome open reading frames (ORFs) comprise only 10\% to $30 \%$ of total metagenome, and are poorly represented in the dataset due to overall low coverage of metagenomic gene pool, even in large-scale projects.

Results: By combining secretome-selective phage display and next-generation sequencing, we focused the sequence analysis of complex rumen microbial community on the metasecretome component of the metagenome. This approach achieved high enrichment ( 29 fold) of secreted fibrolytic enzymes from the plant-adherent microbial community of the bovine rumen. In particular, we identified hundreds of heretofore rare modules belonging to cellulosomes, cell-surface complexes specialised for recognition and degradation of the plant fibre.

Conclusions: As a method, metasecretome phage display combined with next-generation sequencing has a power to sample the diversity of low-abundance surface and secreted proteins that would otherwise require exceptionally large metagenomic sequencing projects. As a resource, metasecretome display library backed by the dataset obtained by next-generation sequencing is ready for i) affinity selection by standard phage display methodology and ii) easy purification of displayed proteins as part of the virion for individual functional analysis.
\end{abstract}

Keywords: Phage display, Nxt generation sequencing, Metagenomics, Rumen, Cellulosome, Surface and secreted proteins

\section{Background}

Microorganisms account for a major proportion of our planet's biological diversity and thus present an enormous and largely unknown resource that can be utilised in the discovery of novel genes, bioactive molecules [1] and new biocatalysts. These may be exploited to improve industrially relevant processes [2]. The traditional approach to tap into this resource is via the cultivation of microorganisms and screening for individual strains with the desired phenotype(s). However, more than $90 \%$ of

\footnotetext{
*Correspondence: j.rakonjac@massey.ac.nz; dragana.gagic@agresearch.co.nz ${ }^{2}$ Institute of Fundamental Sciences, Massey University, Palmerston North 4442, New Zealand

${ }^{1}$ Animal Nutrition and Health, AgResearch Ltd, Palmerston North 4442, New Zealand

Full list of author information is available at the end of the article
}

microbes in complex microbial communities are not culturable by standard laboratory techniques [3]. The nature of these complex microbial communities is being realised in culture-independent approaches, collectively known as metagenomics [4]. These approaches range from the amplification and deep sequencing of phylogenetically informative genes and regions within community DNA (such as the 16S rRNA gene) to assess community structure, shotgun sequencing of community DNA to determine their coding potential, through to targeted functional screens of libraries constructed from community DNA [5-7].

The fermentative forestomach of ruminant animals, known as the reticulo-rumen, is one of the most complex microbial ecosystems investigated via metagenomic studies [8]. Since the 1980s, the rumen has been used as a 
source for the discovery of enzymatic activities involved in the degradation of the lignocellulosic components of the plant cell wall for both agricultural and biofuel production applications [9-11]. It is estimated that the rumen harbours up to 3,000 bacterial species, the majority belonging to the phyla Firmicutes and Bacteroidetes, with species belonging to the Proteobacteria, Fibrobacteres and Spirochaetes also present [12-15].

Rumen microorganisms metabolise plant structural carbohydrates using a broad spectrum of Carbohydrate-Active enZymes, commonly known as CAZymes [16,17], including glycoside hydrolases (GHs), carbohydrate esterases (CEs), glycosyltransferases (GTs) and polysaccharide lyases (PLs). Many CAZymes are modular, containing one or more catalytic domain(s) and ancillary non-catalytic modules including carbohydrate binding modules (CBMs). CBMs are thought to increase the efficiency and specificity of the catalytic module by attachment to a specific sugar moiety [18-20]. A feature of some rumen microbes is the association of CAZymes with cell wall-bound multienzyme structures called cellulosomes $[21,22]$. Cellulosomal CAZymes contain signature domains (dockerins) that anchor the enzymes to cognate domains (cohesins), of a bacterial envelope-bound scaffold composed of one or more proteins called scaffoldins [23]. The synergistic action of CAZymes that assemble as cellulosomes is usually associated with improved fibrolytic function, rendering these surface complexes a desirable target for identification and functional characterisation [24,25].

Secreted CAZymes, including the non-catalytic cellulosome components (e.g. scaffoldins), are but a small fraction of the surface and secreted proteins that make up the "secretome" of a microbial community (metasecretome) [26-29]. Proteomics, despite its power in analysing watersoluble proteins, allows a very limited detection of cellsurface and membrane proteins. Furthermore, at the scale of microbial communities, proteomic approaches are highly dependent on the preparation method and only detect the most abundant secreted or membrane proteins, with the low-abundant proteins escaping identification [30,31]. Most secretome proteins have membranetargeting signal sequences and transmembrane $\alpha$-helices, including the classical Type I, Type II lipoprotein, Type IV prepillin and the twin arginine translocon (Tat) signal sequences [32]. These sequences can be used to predict secretome proteins from sequenced genomes using various algorithms (e.g. SignalP [33], SecretomeP [34], TMHMM [35], and PRED-LIPO [36]). Despite the ability to predict metasecretome proteins in silico, direct analysis of metasecretome proteins (whose coding sequences are predicted to comprise $10-30 \%$ of total ORFs within the metagenome) is desirable to confirm their functions [14,37-39].

Recently, phage display technology has been adapted for the direct selection and display of secretome proteins, and was applied at a single genome scale to Lactobacillus rhamnosus and Mycobacterium tuberculosis [40,41]. Sequence analysis and affinity screenings of the resulting phage display secretome libraries allowed characterisation of surface proteins with functions of interest [40-43]. This technology has potential application at a scale of an entire microbial community, where cultivationindependent methods are required to enable discovery and functional characterisation of products encoded by complex microbial communities. Phage display allows affinity screening of large libraries for functions of interest due to the physical connection of the displayed proteins to the phage-encapsidated coding nucleic acid; displayed proteins can also be easily purified as part of the virion [44-46]. However, given that the published secretome-selective phage display system is limited by the $E$. coli inner membrane translocation systems for the display of secretome proteins, it was uncertain whether this method would limit the diversity of displayed secretome proteins from the taxonomically diverse species that constitute the rumen microbial community.

In this study we applied the secretome-selective phage display method at a metagenomic scale, in combination with next-generation sequencing, and showed that it efficiently displayed functionally and taxonomically diverse secretome proteins, further focusing sequencing effort onto a subset of biologically relevant sequences from a very complex microbial community. In doing so, this approach permitted the discovery of a large assortment of new secreted CAZymes from the bovine rumen microbial community, in particular, expanding the known diversity of cellulosome components, likely to be involved in ruminal fibre degradation.

\section{Results}

\section{Efficiency of metasecretome phage display library} selection, secretion signals and phylogenetic diversity

A shot-gun library was constructed in a phagemid/helper phage secretome-selective phage system as described in Jankovic et al. [40] (see Figure 1 for schematic overview of library construction). To maximise the probability of identifying extracellular proteins involved in fibre degradation, a plant-adherent fraction of the rumen microbial community from pasture-fed cows was used as a source of DNA for library construction. A small pilot library was initially constructed in the secretome-selection phagemid vector pDJ01 [40]. The primary size of this library (before secretome selection) was $4 \times 10^{5}$ clones, and the insert size range was approximately 0.7 to $5 \mathrm{~kb}$. The library was subjected to secretome selection, producing a recombinant clone pool enriched for secretome proteins, in the form of recombinant phagemid single stranded DNA (ssDNA) [40]. To assess the efficiency of selection, ssDNA was transformed into E. coli TG1 and 90 individual transformants were analysed by sequencing the phagemid inserts. 


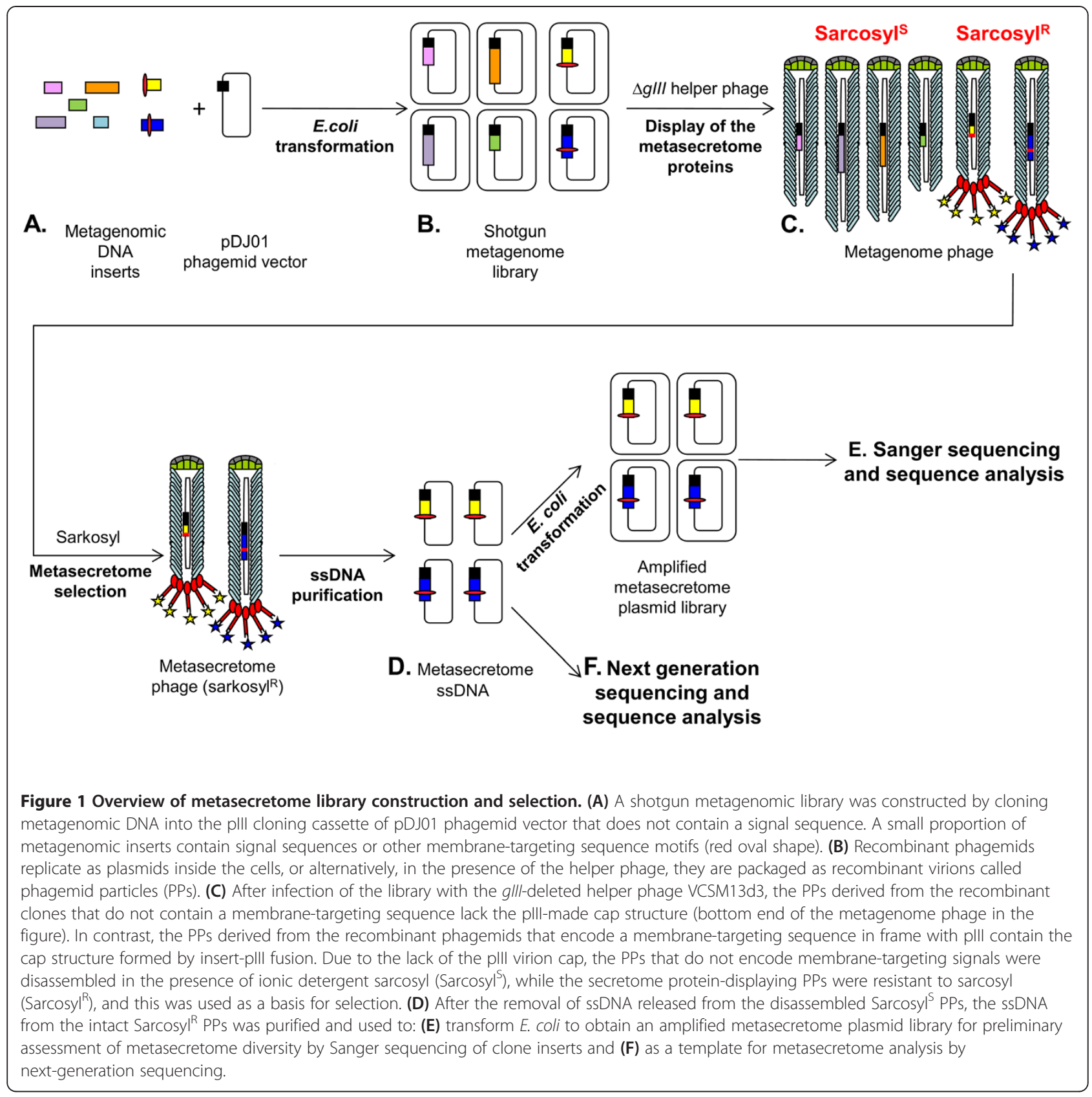

It was found that 85 of the 90 inserts analysed (94.4\%) contained 53 distinct ORFs encoding secretome proteins with typical signal sequences in-frame with pIII. Of the remaining five inserts (5.6\%), one contained an ORF encoding a polypeptide in frame with pIII that was shorter than 24 amino acid residues and was considered "background" (Figure 2). The remaining four inserts contained a single ORF without typical membrane-targeting sequence. Further analysis using SecretomeP 2.0, which discriminates between non-classically secreted proteins and cellular proteins based on amino acid composition, secondary structure and disordered regions, gave score
$<0.5$, which indicates that polypeptide encoded by this ORF is not secreted via non-classical secretion pathways. BLAST analysis was used to predict localisation of the putative protein based on sequence homology. The protein showed homology to a conserved hypothetical protein with predicted cytoplasmic localisation, and was therefore also considered "background" that was not eliminated by selection (Figure 2).

Based on the average proportion of secretome ORFs in bacterial genomes $(\sim 20 \%)$, and the probability of the insert being in the same orientation (50\%), and in-frame (33.3\%) with gene $g I I I$ to create an in-frame protein 


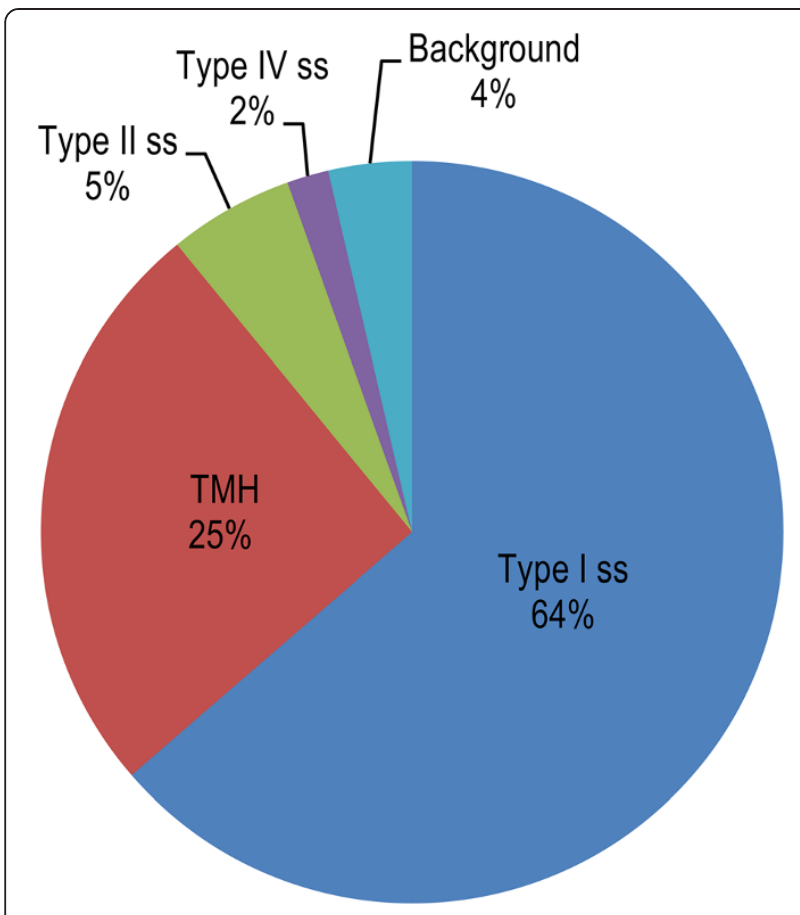

Figure 2 Types of membrane-targeting signals detected in metasecretome pilot library ORFs. Abbreviations used for membrane-targeting signal types: ss, signal sequence; Type I ss, classical ss; Type II ss, lipoprotein ss; Type IV ss, pilin-like ss; TMH, Nterminal or internal transmembrane a helix/helices; background ORFs without membrane-targeting signal or shorter than 24 amino acids.

fusion with pIII, we expect only $\sim 3.3 \%$ of the inserts in the library to be selected. Therefore, the efficiency of selection was estimated by comparing the frequency of secretome insert-containing recombinant phagemids after selection $85 / 90$ (94.4\%) with the theoretically predicted frequency (3.3\%). The enrichment of the secretome insert-containing recombinant library clones was 29-fold, indicating that the stringency of selection was high, and that most recombinant phagemids containing non-secretome inserts (background) were eliminated.

The types of membrane-targeting signals predicted from the pilot metasecretome phage display library ORFs are summarised in Figure 2, while the membrane-targeting sequences and detailed analysis are presented in Additional file 1 . The majority of ORFs (35) contained type I signal sequences while the remainder consisted of transmembrane $\alpha$-helices with $\mathrm{N}$-terminal transmembrane anchors (8), multiple transmembrane $\alpha$-helices or single internal transmembrane $\alpha$-helices (6), type II or lipoprotein signal sequences (predicted in three ORFs), and a single type IV (pillin-like) signal sequence. Selection of protein-pIII fusions containing type II signal sequences or transmembrane helices has been observed in genomic secretome- selective display [40], despite the fact that the native pIII signal sequence is type I. It appears that a predicted transmembrane $\alpha$-helix and dependence on the SecYEG translocon is the condition for assembly of sarcosyl-resistant recombinant virions. The absence of the Tat signal sequences likely stems from the fact that their export depends on the specific TatABC translocon, involved in the transport of folded substrates. It was shown that Tat pathway is not suitable for targeting of the pIII fusions to the virion, since protein-pIII fusion typically folds in the oxidising environment of the E. coli periplasm, in contrast to the Tat-dependent proteins that fold in the reducing environment of the cytoplasm $[47,48]$.

To identify the organisms from which metasecretome clones were derived, taxonomic assignments were designated for the predicted proteins of each insert, based on the best BLASTX hits, where the E-value was less than $1 \times 10^{-5}$ and query coverage greater than $30 \%$. The most abundant assignments were to the genera Prevotella (13\%), Clostridium (10\%), Butyrivibrio (7\%), Ruminococcus (6\%), Bacteroides (6\%) and Fibrobacter (4\%); genus-level assignments could not be made for $50 \%$ of the inserts analysed. These results indicate that the metasecretome selection method captured representatives of the main genera comprising the core bovine rumen microbiome, as previously determined by pyrosequencing of $16 \mathrm{~S}$ rRNA genes of other rumen microbial communities [15,49].

\section{Metasecretome characterisation by next-generation sequencing}

The small scale of the pilot metagenome library and metasecretome selection that included transformation bottleneck and standard Sanger sequencing did not allow access to the large diversity of the rumen microbial metasecretome. Therefore, to improve on the representation of the metasecretome, an upscaled primary metagenomic library was constructed with a final size (before selection) of $\sim 5 \times 10^{6}$ primary clones. Furthermore, the secretome selection protocol was combined with the next-generation sequencing of inserts. After secretome selection [40], the inserts from the resulting metasecretome ssDNA pool were PCRamplified and processed by enzymatic and mechanical shearing to fragments of a suitable size range (600 - $800 \mathrm{bp}$ ) for 454 GS FLX sequencing. A total of 691,206 obtained sequence reads were obtained and processed (including trimming, low complexity filtering and de-replication), resulting in 153,002 de-replicated reads that were further analysed (see Additional file 2 for the NGS summary and statistics).

To predict the putative functions that were enriched in the metasecretome library, the metasecretome sequence data was compared to a 454 GS FLX shotgun sequenced metagenome derived from the plant-adherent rumen microbial fraction of two New Zealand cows grazing a similar pasture-based diet (data not published). Annotation 
of the metasecretome and metagenome sequence reads via IMG/M system [50] resulted in 35\% and 49\% Pfam [51] assignments of the total protein coding genes, respectively, which were further categorised into COGbased functional categories (Figure 3). The functional category with the most assignments was "carbohydrate transport and metabolism" for both the metagenome (10.6\%) and the metasecretome datasets (19.4\%) (Figure 3, bar G). Metasecretome phage display also enabled enrichment of proteins predicted to be involved in the "cell wall/membrane/envelope biogenesis" (Figure 3, bar M) and peptides with unknown function (Figure 3, bar S). Proteins of unknown function are generally overrepresented in the secretome fraction of bacterial genomes $[52,53]$, and their enrichment is consistent with enrichment of the metasecretome. In contrast, the functional categories of "replication, recombination and repair" (Figure 3, bar L) and "coenzyme transport and metabolism" (Figure 3, bar $\mathrm{H}$ ), comprised mainly of intracellular proteins, were under-represented in the metasecretome dataset.

\section{Carbohydrate-active enzyme (CAZyme) diversity and abundance of cellulosome components within the metasecretome selected library}

The metasecretome (and metagenome) ORFs were analysed using the dbCAN database to determine the diversity of CAZyme families captured by the metasecretome selection (Table 1). The dbCAN database uses Hidden Markov Models (HMMs) of the signature domain regions for all CAZyme families, and incorporates the most

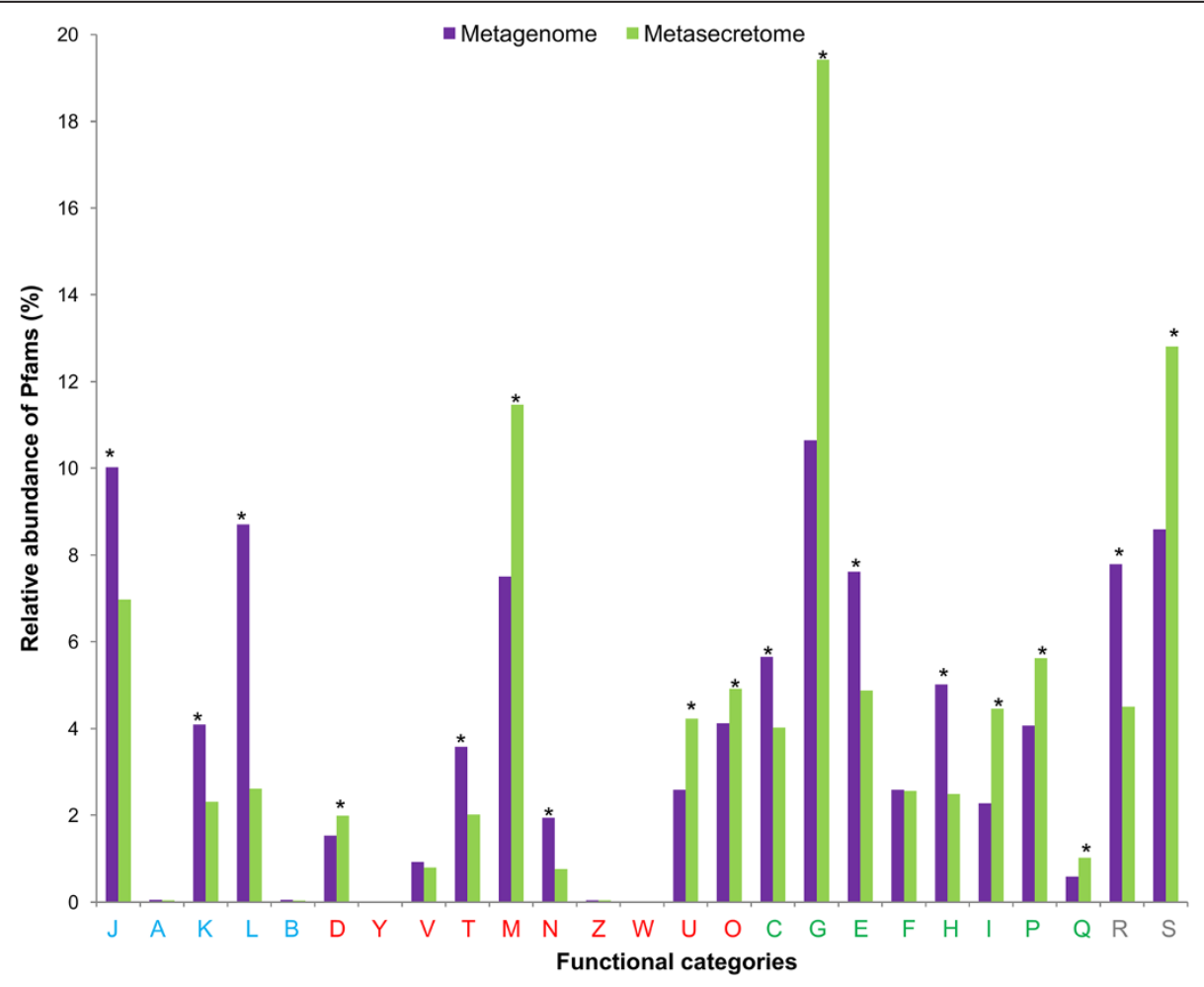

Figure 3 Relative abundances of Pfams within the metagenome and metasecretome-enriched sequence datasets. Relative abundances of IMG/M annotated COG-based functional categories of protein family (Pfam) conserved domains within the metagenome (purple bars) and metasecretome-enriched (green bars) sequence datasets. Abbreviations for the functional categories, grouped by general functional role: Information storage and processing (blue font): J - Translation, ribosomal structure and biogenesis, A - RNA processing and modification, $\mathrm{K}$ - Transcription, L - Replication, recombination and repair, B - Chromatin structure and dynamics; Cellular processes and signalling (red font): D - Cell cycle control, cell division, chromosome partitioning, $Y$ - Nuclear structure, $V$ - Defence mechanisms, $T$ - Signal transduction mechanisms, M - Cell wall/membrane/envelope biogenesis, N - Cell motility, Z - Cytoskeleton, W - Extracellular structures, U - Intracellular trafficking, secretion and vesicular transport, $\mathrm{O}$ - Posttranslational modification, protein turnover, chaperones; Metabolism (green font): $\mathrm{C}$ Energy production and conversion, G - Carbohydrate transport and metabolism, E - Amino acid transport and metabolism, F - Nucleotide transport and metabolism, $\mathrm{H}$ - Coenzyme transport and metabolism, I - Lipid transport and metabolism, P - Inorganic ion transport and metabolism, Q - Secondary metabolites biosynthesis, transport and catabolism; Poorly characterized (grey font): R - General function prediction only, S - Function unknown. Significant difference between metasecretome and metagenome datasets within given functional category is represented by asterisks ( $P \leq 0.001)$. 
Table 1 Comparison of CAZyme classes between plant-adherent rumen microbial metasecretome and metagenome datasets

\begin{tabular}{|c|c|c|c|c|}
\hline CAZyme class & Count MS & Distribution MS & Count MG & Distribution MG \\
\hline Carbohydrate-binding modules & 1038 & $8.3 \%$ & cpo1656 & $7.6 \%$ \\
\hline Carbohydrate esterases & 1499 & $11.9 \%$ & 2235 & $10.2 \%$ \\
\hline Glycoside hydrolases & 7639 & $60.8 \%$ & 11606 & $53.2 \%$ \\
\hline Glycosyl transferases & 793 & $6.3 \%$ & 5126 & $23.5 \%$ \\
\hline Polysaccharide lyases & 382 & $3.0 \%$ & 524 & $2.4 \%$ \\
\hline Auxiliary activities & 67 & $0.5 \%$ & 451 & $2.1 \%$ \\
\hline Cellulosome components* & $1147(577)$ & $9.2 \%(7.2 \%)$ & $225(207)$ & $1.0 \%(0.9 \%)$ \\
\hline SLH & $46(34)$ & $0.37 \%(0.43 \%)$ & $77(72)$ & $0.35 \%(0.33 \%)$ \\
\hline cohesins & $52(44)$ & $0.41 \%(0.55 \%)$ & $27(27)$ & $0.12 \%(0.12 \%)$ \\
\hline dockerins & 1049 (499) & $8.35 \%(6.25 \%)$ & $121(108)$ & $0.55 \%(0.50 \%)$ \\
\hline Total ${ }^{*}$ & $12565(7978)$ & $100.0 \%$ & $21823(21607)$ & $100.0 \%$ \\
\hline
\end{tabular}

Abbreviations: MS, metasecretome dataset; MG, metagenome dataset. *Numbers in parentheses refer to the CAZYme hits clustered at $100 \%$ sequence identity to remove duplicity and were used in analysis of cellulosome hit frequencies.

complete set of metagenomic CAZyme genes published so far [54]. The analysis identified 12,565 putative CAZyme hits in the metasecretome library with a significant match to at least one catalytic domain or associated module belonging to 196 different CAZy families while the analysis of metagenome $(21,823$ hits) identified 318 CAZy families (Additional file 3).

In both datasets we captured an assortment of cellulases, endoxylanases, carbohydrate debranching enzymes and oligosaccharide-degrading enzymes, as well as a suite of carbohydrate esterases responsible for deacetylation of xylans and xylo-oligosaccharides, and polysaccharide lyases. The GH profile of the metasecretome dataset was also similar to other reported bovine metagenomes except that GH53 (exclusive $\beta$-1,4-galactanase), responsible for degradation of galactans and arabinogalactans, and GH43 (various oligosaccharide degrading enzymes) were detected in abundance $[13,14]$. When compared to the control metagenome dataset, xyloglucanases GH16 and GH74, and other oligosaccharide degrading enzymes belonging to $\mathrm{GH} 2$ and $\mathrm{GH} 3$ families occurred at higher frequency in the metasecretome dataset. In contrast, endohemicellulases (GH8, GH10) and debranching enzymes (GH51, GH67, GH78) occurred at lower frequency in the metasecretome dataset. Other GH class members that were enriched and significantly more abundant in the metasecretome compared to the metagenome dataset belong to families GH124 (cellulosomal endoglucanases; 14.3-fold enrichment), GH55 ( $\beta$-1,3-glucanases; 6.5 -fold) and GH92 ( $\alpha$-mannosidases; 5.9-fold). In the CAZy database, GH family 124 has only one characterised enzyme while a prokaryotic representative of $\mathrm{GH}$ family 55 has not been yet characterised. The CBMs prevalent in metasecretome, CBM67 and CBM40, are usually associated with catalytic modules of GH78 and GH33; however, representatives of these $\mathrm{GH}$ families were not found in large numbers in this dataset. In concordance with their extracellular function, several CE families involved in hemicellulose (CE1, CE3, CE7) and pectin (CE8) degradation detected in metasecretome were enriched and significantly more abundant than in the metagenome. The analysis of glycosyl transferases (GTs), the enzymes that assemble glycans (glycoproteins, glycolipids, oligosaccharides), showed a decrease from $23.5 \%$ in the metagenome to $6.3 \%$ in the metasecretome, consistent with the evidence that the majority of bacterial GTs are located in the cytoplasm [55].

A high number of putative components [cohesins, dockerins and surface layer homology (SLH) modules] of complex carbohydrate-degrading surface complexes cellulosomes were detected (Figure 4). Analysis of metasecretome ORFs with hits to cellulosome-associated modules, clustered at $100 \%$ sequence identity to remove duplicity, revealed that $6.3 \%$ of the total clustered CAZyme hits were to dockerins (Table 1). Of those, $4.5 \%$ hits were to a HMM representing a single dockerin repeat; $1.7 \%$ were to presumably complete dockerin domains (containing two hits to dockerin repeat HMMs) and $0.1 \%$ were to single dockerin repeat in combination with another CAZyme module. Two other modules present in cellulosomes, cohesin and SLH, were also detected ( $0.6 \%$ and $0.4 \%$, respectively).

The phylogenetic diversity of the translated CAZyme ORFs predicted to contain cellulosome modules was determined by family-level taxonomic assignment based on the best BLASTP hit (Figure 5), and the recently proposed reclassification of Clostridium spp. based on extensive molecular phylogenetic data [56,57]. Around two thirds of cohesin modules containing sequences were assigned to the Firmicutes [including Ruminococcaceae $(40 \%)$ and Eubacteriaceae (25\%)], with the remaining 


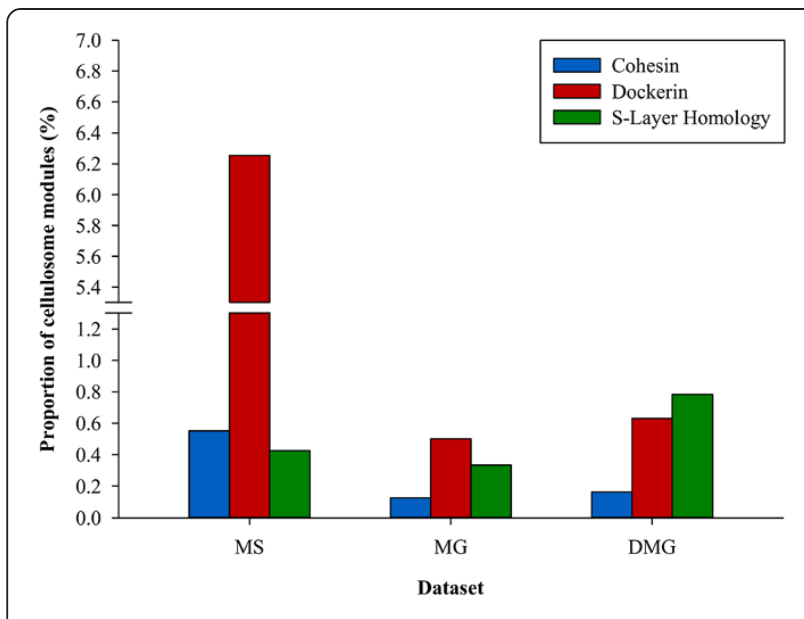

Figure 4 Frequency of cellulosome modules in three bovine rumen microbial datasets. Frequency of three cellulosome signature modules: cohesin (blue); dockerin (red) and surface layer homology (SLH) domains (green) were compared between three datasets: MS, metasecretome; MG, metagenome (both derived from the plant-adherent rumen microbial community fraction isolated from fistulated pasture-grazing dairy cows) and DMG, published deep-sequenced metagenome dataset derived from the bovine switchgrass-adherent microbiome, isolated from switchgrass that was incubated in the rumen of a fistulated cow for $72 \mathrm{~h} \mathrm{[14].} \mathrm{The}$ total number of distinct CAZyme hits, obtained after clustering all dbCAN hits at 100\% sequence identity threshold using the CD-HIT algorithm [76], were: MS, 7,978; MG, 21,607; DMG, 123,223.

assigned to Bacteroidetes [Flavobacteriaceae (20\%) and Bacteroidaceae (10\%)]. The vast majority of dockerincontaining sequences were assigned to the Firmicutes [including Ruminococcaceae (61\%) and Clostridiaceae (17\%)] and Bacteroidetes representation was mainly within the Bacteroidaceae (7.3\%), and Prevotellaceae (2.9\%). Among the best BLASTP hits, many were to species that have been previously reported as cellulosome-producers, such as Acetivibrio cellulolyticus, Clostridium acetobutylicum, Ruminococcus albus, R. flavefaciens, Ruminiclostridium cellulolyticum (formerly Clostridium cellulolyticum), $R u$. josui (formerly C. josui) and Ru. thermocellum (formerly C. thermocellum) [22]. In contrast, 97\% of putative SLH domains were assigned to Firmicutes (including 53\% to Lachnospiraceae, $29 \%$ to Veillonellaceae and $15 \%$ to Ruminococcaceae).

\section{Phylogenetic diversity of the selected metasecretome}

We used an IMG/M similarity-based binning approach for the taxonomic assignment of the predicted proteincoding sequences, and to determine their phylogenetic distribution (Figure 6). The majority of assigned sequences belong to Bacteria (40.9\%), $0.2 \%$ to Archaea and $0.1 \%$ to Eukaryota, while $58.8 \%$ remained unassigned. Approximately $28 \%$ of the sequences assigned to Eukaryota were most similar to fungi and around $14 \%$ to plants, which may reflect the presence of low levels of plant and fungal material within our plant-adherent microbiome samples. Virus hits were rare $(0.004 \%)$. At the phylum level, Bacteroidetes (29\%) and Firmicutes (10\%) dominated, with minor contributions from Proteobacteria, Actinobacteria, Spirochaetes and Cyanobacteria. The main taxonomic assignments are in agreement with predominant phyla determined in the 16S rRNA gene based studies of bacterial diversity of other rumen microbial communities [15]. A higher representation of sequences from Gram-negative bacteria was apparent in the metasecretome dataset relative to the metagenome dataset. This was consistent with taxonomic representation of the metasecretome pilot library inserts, and might be due to a somewhat higher efficiency of Gram-negative relative to Gram-positive membrane-targeting signals in E. coli as a host strain.

\section{Discussion}

Improving the digestive processes of ruminant animals, or degradation of lignocellulosic feedstocks for biofuel production, requires an understanding of the enzymatic processes involved in the depolymerisation of plant structural carbohydrates. The majority of the information currently available has been generated from the study of individual microbes and their enzyme complements, but in nature the breakdown of plant polysaccharides is initiated by microbial consortia and their secreted enzymes. This is much more complex and difficult to study, but the recent development of high-throughput sequencing and associated metagenomic techniques opens up new opportunities to begin to understand this complex process. In this study we have assessed the rumen metasecretome, using a secretome-selective phage display technology that enables the focusing of next-generation sequence analysis to this portion of the metagenome. This is, to our knowledge, the first report of selective sequence analysis as a method to focus on the sequences encoding secreted proteins from a metagenome. The rumen microbial metasecretome is specialised for the initial degradation of plant fibre through the action of surface-associated and secreted enzymes. Consistent with this, the metasecretome display approach has considerably enriched for secretome proteins in the "carbohydrate transport and metabolism" functional category. This functional category was represented in the metasecretome dataset with a wide diversity of GH catalytic modules, assigned to $85 \mathrm{GH}$ families, accompanied by a variety of CBMs (belonging to 38 CBM families), CEs (13 families) and PLs (10 families).

The selectivity of the method was apparent when the abundance of two subcategories of CAZymes: GTs and cellulosomal modules (specifically, cohesins and dockerins) 


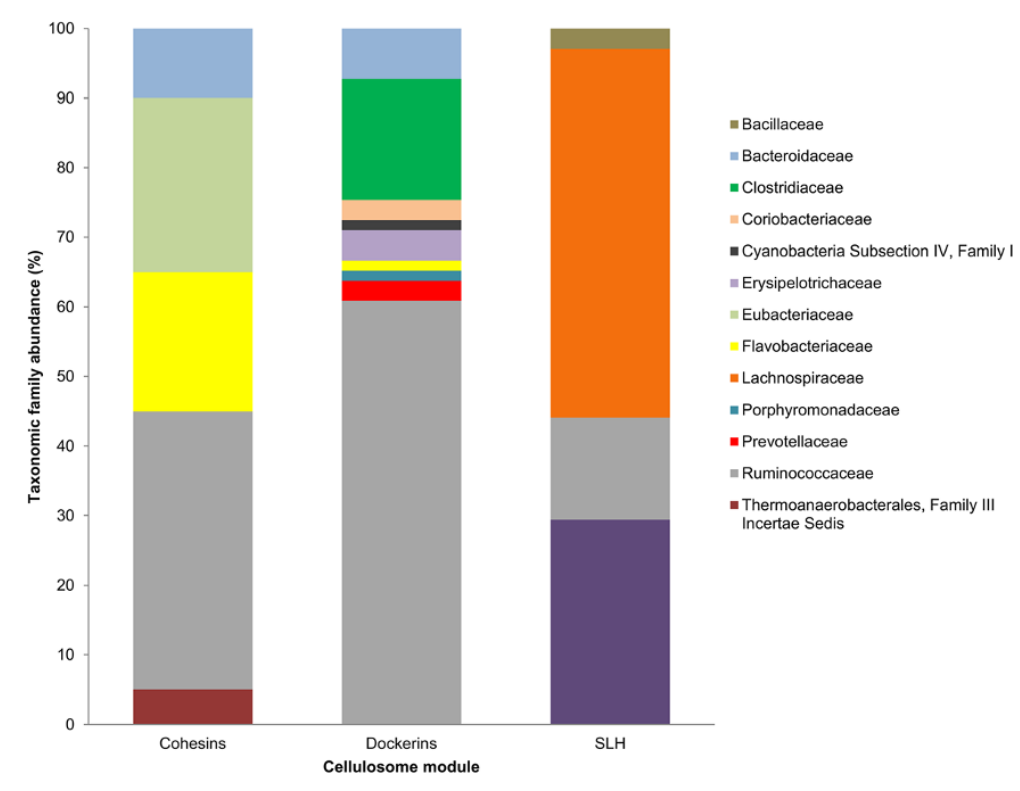

Figure 5 Phylogenetic diversity of cellulosome modules predicted in the rumen metasecretome-enriched dataset. Translated metasecretome ORFs that were predicted to contain cellulosome modules (cohesin, dockerin and SLH domains) were compared to the nonredundant protein database using BLASTP. Family-level taxonomic assignments were made for the host organism of the best BLAST hit and the chart shows the abundance of each family for each cellulosome module. For the dockerin data, only sequences that contained two dockerin modules $(\mathrm{N}=69)$ are shown.

were compared to corresponding groups in a metagenome dataset. The relatively lower representation of GTs in the metasecretome is consistent with the current knowledge of GTs cytosolic localisation in bacteria [58]. On the other hand, proteins containing cohesin and dockerin domains are secreted or membrane-bound, as described for several anaerobic bacteria, notably $R u$. thermocellum and C. cellulovorans, and R. flavefaciens FD1 [58-60]. A striking difference in comparison with reports from previous rumen microbiome studies and our metagenome lies in the presence of a high frequency of putative cohesin and dockerin modules. For example, comparison of the abundance of cellulosome-associated modules in our metasecretome dataset, with those in a switchgrass-adherent bovine rumen microbial metagenomic sequence dataset [14], predicted using the same database and search parameters [54], showed a prominent enrichment for cohesin and dockerin modules (Figure 4). Other published rumen metagenomic datasets have detected even lower proportions of cellulosomal modules $[13,61,62]$. The majority of the metasecretome inserts predicted to encode dockerin and cohesin modules showed strong homology to sequences from members of the Ruminococcaceae [56]. This finding is reasonably consistent with the taxonomic affiliations of known cultivated cellulosome producing-bacteria, which are also predominantly from the Ruminococcaceae [22].
Our results suggest that, within the plant-adherent rumen microbial fraction, members of the Ruminococcaceae also have the greatest potential to produce cellulosomelike structures. A number of cohesin (10\%) and dockerin (7.25\%) containing inserts were assigned to the Bacteroidaceae, suggesting potential for this family to produce cellulosomes. However, currently there are no reports of cellulosome-producing organisms from this family. Interestingly, one of the earliest reported cellulosome producers, Bacteroides cellulosolvens [63], is now recognised as a member of the Ruminococcaceae where it has been reclassified as Ruminiclostridium cellulosolvens [56]. In the metasecretome dataset, almost $18 \%$ of the dockerinencoding inserts were most similar to sequences from members of the Clostridiaceae, although curiously, cohesincontaining ORFs that are also associated with this family were not detected. In total, only 44 sequences with hits to cohesin domains were detected in this study, as compared to more than 400 predicted dockerin-containing sequences. Within the genomes of cellulosome-producing organisms, scaffoldin genes encoding cohesin domains are not nearly as abundant as those encoding dockerin motifs, thus we may have simply missed capturing the cognate Clostridiaceae-derived cohesin encoding genes by chance. At 168 amino acid residues, the cohesin HMM is longer than that for a dockerin repeat (22 residues) within dbCAN. Therefore, with metasecretome 


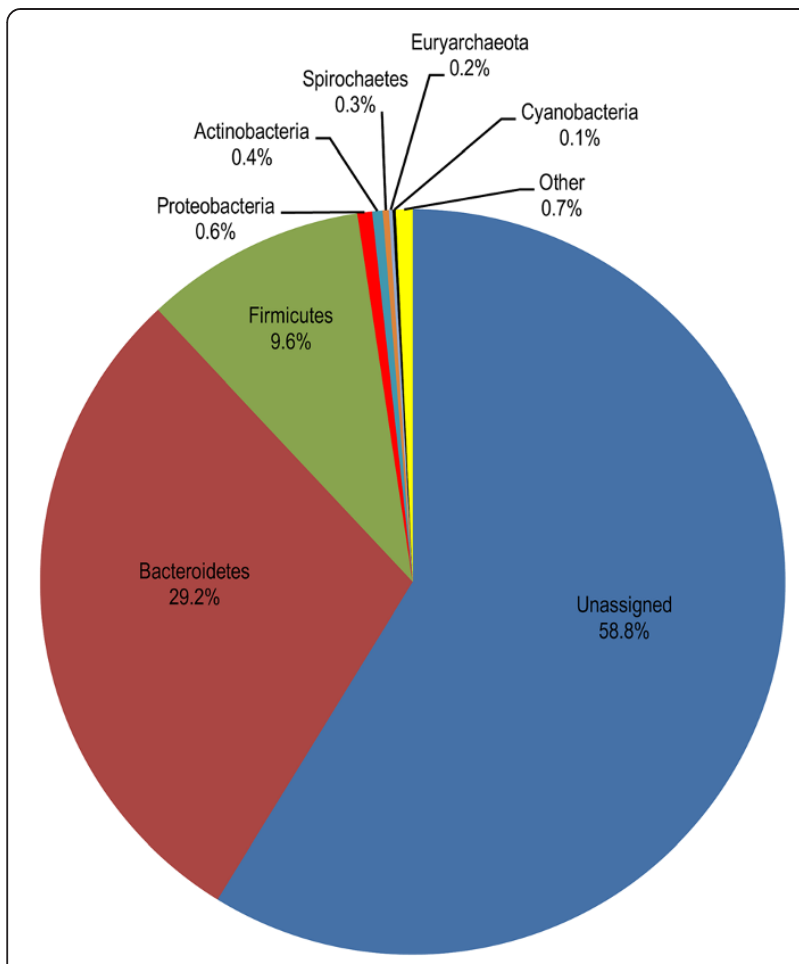

Figure 6 Phylogenetic profile of the metasecretome-enriched dataset. The taxonomic assignment of the metasecretome reads derived from the rumen adherent microbial fraction was based on distribution of best BLAST hits of protein-coding genes at 30\% BLAST identity. Slices of a pie chart are corresponding to the percentage of total best BLAST hits at phyla level. The "Other" contains ORFs with database hits belonging to a phylogenetic group of low abundance in the dataset $(<0.1 \%)$, while the "Unassigned" corresponds to predicted ORFs with hits below 30\% identity cut-off.

library inserts being generally small in size, partial capture of cohesin sequences may not have enabled their in silico detection. Moreover, in case of $R$. albus strain 8, a putative cellulosome producer with many genes predicted to encode dockerin-containing enzymes for which putative cohesin domain-encoding genes have not been yet identified, it was speculated that closely related rumen bacteria may produce cognate cohesin-bearing scaffoldins that could enable appropriation of the dockerin-containing enzymes produced by $R$. albus 8 [22].

A small number of dockerin and cohesin modulecontaining sequences appeared to be associated with a number of bacterial families that are not known to produce cellulosomes, such as the Coriobacteriaceae, Erysipelotrichaceae and Porphyromonadaceae. It is thus uncertain whether these are from cellulosome-producing organisms. Alternatively, they may be associated with proteins that mediate roles in interactions that are not involved in cellulosomal function, but rather in proteolysis (proteases), oxidative reduction (peroxidases) or dephosphorylation (phosphatases) [64]. It has been hypothesised that in the complex ecosystems different organisms could use cohesin and dockerin modules to interact in a form of intespecies cell-cell adhesion. Alternatively, these proteins may evolve to attain different roles unrelated to cell-adhesion [64].

\section{Conclusions}

The metasecretome phage display method combined with next-generation sequencing has the power to functionally select for, and reveal, the diversity of low-abundance surface and secreted proteins that would otherwise require large metagenomic sequencing efforts to reveal. This approach allowed the identification of a large number of cellulosomal module-containing proteins and produced a rumen microbial metasecretome display library that is currently being used to explore the roles of rumen bacterial cellulosomes and other CAZymes via standard phage display affinity selection and protein display methodologies. The novel CAZyme genes and domains identified from this study represent valuable candidates for further analysis, starting from the metasecretome library as a resource. For example, interacting pairs of cohesins and dockerins could be determined by affinity-panning of the metasecretome library using expressed cohesins as baits, whereas carbohydrate binding modules of interest could be identified by screening the metasecretome library using the complex carbohydrates as baits. Furthermore, screening of the protein repertoire displayed on the surface of metasecretome library virions for novel biocatalysts of interest $[65,66]$, using the reaction product-based trapping strategies or by colony-based colorimetric detection, could be used to explore the enzymatic activities that could be potentially exploited in industrial processes involving fibre degradation.

\section{Methods}

\section{Rumen sampling and rumen content fractionation}

A sample of whole rumen content was obtained from a fistulated Friesian dairy cow, grazing ad libitum on a ryegrass - clover pasture diet, supplemented with pasture silage $(\sim 10 \%$ of the recommended daily intake per animal). The sampling was conducted in May 2009 at Lye Farm, DairyNZ (Waikato, New Zealand) under the animal ethics permission number AE 11483 granted by the Ruakura Animal Ethics Committee. Between 1 and $1.5 \mathrm{~kg}$ of rumen contents was collected in the morning and immediately processed. A protocol for partitioning of the rumen microbial fraction tightly adherent to plant biomass (plant-adherent fraction) from liquid (planktonic) and associated (loosely attached) microbial fractions is described in detail in Additional file 4. Fractions and samples of digesta obtained from different phases of the process were snap-frozen in liquid nitrogen and kept on dry ice until long term storage at $-80^{\circ} \mathrm{C}$. 
Bacterial strains, display system and growth conditions Escherichia coli strain TG1 (supE thi-1 $\Delta($ lac-proAB) $\Delta($ mcrB-hsdSM $) 5\left(\mathrm{r}_{\mathrm{K}}^{-} \mathrm{m}_{\mathrm{K}}^{-}\right)$[F' traD36 proAB lacl $\left.\left.{ }^{\mathrm{q}} Z \Delta M 15\right]\right)$ was used as a host for the construction of phage display libraries, as well as for propagation of the wild-type helper phage, VCSM13 (Stratagene, USA). The E. coli strain K1976 (TG1 transformed with plasmid pJARA112 that expresses gIII under the control of phage-inducible promoter ppsp) was used to obtain infectious stocks of the helper phage VCSM13d3, containing deletion of the complete gIII coding sequence [67].

Phagemid vector pDJ01 [40], designed for selective secretome display, was used for construction of the metasecretome libraries. The display cassette of pDJ01 contains the promoter $\mathrm{p} p s p$, followed by the ribosome-binding site, the start (ATG) codon, multiple cloning site and the sequence encoding the $\mathrm{C}$-domain of phage protein pIII. In contrast to other display vectors, pDJ01 does not have a signal sequence. This vector also contains a chloramphenicol resistance marker $\left(\mathrm{Cm}^{\mathrm{R}}\right)$, plasmid (ColE1) origin of replication, and phage intergenic sequence containing $\mathrm{f} 1$ origin of replication and packaging signal. When helper phage VCSM13d3 is used to assemble phagemid-containing virion particles (PPs), empty pDJ01 vector only produces defective particles that are sensitive to the detergent sarcosyl $[0.1 \%(\mathrm{w} / \mathrm{v})]$. Inserts that contain a signal sequence or other motifs that can mediate targeting the N-terminus of the fusion into the $E$. coli membrane or the periplasm are required for assembly of the pIII C-domain into the virion and formation of detergent-resistant virions ([40]; Figure 1).

E. coli cells were incubated in $2 \times$ Yeast Extract Tryptone broth $(2 \times \mathrm{YT})$ at $37^{\circ} \mathrm{C}$ with aeration $(200 \mathrm{rpm})$. Solid medium for growth of $E$. coli transformants also contained 1.5\% (w/v) bacteriological agar (Oxoid, USA) unless otherwise indicated. When required, antibiotics were added to media at the following concentrations: $25 \mu \mathrm{g} \mathrm{ml}^{-1}$ chloramphenicol $(\mathrm{Cm})$ and $60 \mu \mathrm{g} \mathrm{ml}^{-1}$ ampicillin (Amp).

\section{Metagenomic DNA extraction from rumen microbial community plant-adherent fraction}

High molecular weight metagenomic DNA from the rumen microbial plant-adherent fraction was extracted according to Stein et al. [68] with some modifications. In total, $2 \mathrm{~g}$ of microbial cell pellet from the plant-adherent fraction was split into five samples which were each separately embedded in $0.7 \mathrm{ml}$ of $1 \%$ low-melting-temperature agarose and incubated in a syringe for $10 \mathrm{~min}$ on ice. Samples were extruded into $10 \mathrm{ml}$ of lysis buffer [1\% (w/v) sarcosyl, $0.2 \%(\mathrm{w} / \mathrm{v})$ sodium-deoxycholate, $10 \mathrm{mM}$ Tris- $\mathrm{HCl}$ ( $\mathrm{pH}$ 8.0), $50 \mathrm{mM} \mathrm{NaCl}, 100 \mathrm{mM}$ ethylenediaminetetraacetic acid (EDTA), lysozyme $(1 \mathrm{mg} / \mathrm{ml})]$ and incubated for $2.5 \mathrm{~h}$ at $37^{\circ} \mathrm{C}$, followed by $17 \mathrm{~h}$ incubation in $40 \mathrm{ml}$ ESP buffer $[0.5 \%(\mathrm{w} / \mathrm{v})$ sarcosyl, $20 \mathrm{mM}$ EDTA and $0.013 \mathrm{AU}$ protease (Qiagen, Germany)] at $55^{\circ} \mathrm{C}$ to inactivate nucleases present in the sample. After addition of fresh ESP buffer $(20 \mathrm{ml})$ to each sample and $1 \mathrm{~h}$ incubation at $55^{\circ} \mathrm{C}$, three washes with TE buffer $[10 \mathrm{mM}$ Tris- $\mathrm{HCl}(\mathrm{pH} 8.0)$, $1 \mathrm{mM}$ EDTA] were performed and remaining proteases were inactivated for $15 \mathrm{~min}$ at $70^{\circ} \mathrm{C}$. To digest agarose, samples were incubated overnight at $37^{\circ} \mathrm{C}$ with $15 \mathrm{U}$ of Agar $A C E^{\mathrm{tm}}$ enzyme (Promega, USA). Residual insoluble oligosaccharides were removed by centrifugation and the supernatant, containing crude DNA released from the agarose, was subjected to phenol:chloroform:isoamyl alcohol extraction (25:24:1). After pooling together the five starting samples, metagenomic DNA was concentrated using a $100 \mathrm{kDa}$ cut-off Vivaspin filter device (Sartorius Stedim Biotech, Germany).

\section{Construction of rumen metagenome phage display libraries}

Two shotgun metagenome phage display libraries were constructed: a small pilot library for preliminary assessment of methodology and a large library. Both libraries were constructed from mechanically sheared metagenomic DNA isolated from the rumen plant-adherent microbial fraction and cloned into the secretome-selective phagemid pDJ01 [40] (Figure 1). Around $150 \mu \mathrm{g}$ of high molecular weight metagenomic DNA in $55 \mathrm{mM}$ Tris- $\mathrm{HCl}$ (pH 8.0), $15 \mathrm{mM}$ $\mathrm{MgCl}_{2}, 25 \%$ glycerol was sheared by nebulisation in disposable medical nebulisers by subjecting the sample to a pressure of 10 psi for $1 \mathrm{~min}$, followed by size fractionation, desalting and concentration in $100 \mathrm{kDa}$ cut-off Vivaspin ultra-filtration spin columns (Sartorius Stedim Biotech, Germany). Prior to cloning, the ends of the metagenomic DNA fragments were repaired using an enzyme cocktail containing T4 DNA Polymerase (Roche, Switzerland), Klenow Enzyme (Roche, Switzerland), and OptiKinase ${ }^{\mathrm{TM}}$ (Affymetrix, USA). Next, DNA was purified by phenol: chloroform:isoamyl alcohol (25:24:1) extraction followed by ethanol-precipitation and resuspension in $150 \mu \mathrm{l}$ of $10 \mathrm{mM}$ Tris- $\mathrm{HCl}$ ( $\mathrm{pH}$ 8.0). Approximately $19 \mu \mathrm{g}$ of the endrepaired metagenomic DNA inserts were ligated to 6.5 $\mu \mathrm{g}$ of the vector pDJ01, which was cut using SmaI restriction endonuclease (Roche, Switzerland) and dephosphorylated using rAPid Alkaline Phosphatase (Roche, Switzerland). Ligated DNA was extracted with phenol:chloroform, precipitated and dissolved in $75 \mu \mathrm{l}$ sterile deionised water.

A total of $2 \mu \mathrm{g}$ of ligated metagenomic DNA was electro-transformed into the $E$. coli TG1 electrocompetent cells to obtain the pilot shotgun library, while the rest of the ligation mixture was used in 27 separate transformation reactions to generate a large shotgun library and overcome a problem of promiscuous (fast growing) clones. The resulting 27 transformant samples were also individually processed through the whole 
metasecretome selection procedure and pyrosequencing sample preparation. To estimate primary shotgun library size, aliquots from each transformation were plated on $\mathrm{Cm}$-containing plates. The remaining portion of each transformation mixture was mixed with $9 \mathrm{ml}$ of $2 \times \mathrm{YT}$ broth containing chloramphenicol $\left(2 \times \mathrm{YT} \mathrm{Cm}_{25}\right)$ and incubated for $8 \mathrm{~h}$ at $37^{\circ} \mathrm{C}$ with aeration to amplify the libraries. Amplified library aliquots were frozen at $-80^{\circ} \mathrm{C}$ in $7 \%$ DMSO, apart from $1 \mathrm{ml}$ that was used immediately for the secretome selection.

\section{Selection of secretome-encoding library clones}

A protocol described previously with modifications was used for direct selection of the metasecretome phage display library [40]. In order for a secretome proteinencoding library to be enriched, it had to fulfil two conditions: i) to be translationally fused (i.e. in-frame) with phage protein pIII encoded by the vector; ii) to encode for a membrane-targeting signal, in order to target vector-encoded phage protein pIII (devoid of signal sequence) to the inner membrane of E. coli. When both of these conditions are met, the peptide fused to pIII allows display of the fusion protein on the surface of the virion and complementation of the assembly defect in the $g I I I-$ deletion helper phage VCSM13d3, resulting in detergentresistant virions (phagemid particles). Selection for secretome-encoding inserts is therefore based on treatment of the library, in the form of phagemid particles, that eliminates detergent-sensitive, while preserving the detergent-resistant phagemid particles [40,41]. A $1 \mathrm{ml}$ aliquot of the overnight culture containing amplified primary library clones was used to inoculate $100 \mathrm{ml}$ of $2 \times$ YT $\mathrm{Cm}_{25}$ media. The exponentially growing culture $\left(\mathrm{OD}_{600}=0.2\right)$ was infected with helper phage VCSM13d3 at a multiplicity of infection 50 (50 phage : 1 bacterium) for $1 \mathrm{~h}$ at $37^{\circ} \mathrm{C}$. Infected cells were harvested by centrifugation at 2,600 $\times g$ for $10 \mathrm{~min}$ at room temperature and the resulting pellet was mixed with $40 \mathrm{ml}$ of soft agar [ $2 \times$ YT broth containing $0.6 \%(\mathrm{w} / \mathrm{v})$ molecular biology grade agarose]. Agarose-embedded cells were poured over 16 selective plates $\left(2 \times \mathrm{YT} \mathrm{Cm}_{25}\right.$ plates containing molecular biology grade agarose instead of bacteriological agar) and incubated overnight at $37^{\circ} \mathrm{C}$ [69]. Phagemid particles were extracted from each plate with $5 \mathrm{ml}$ of $2 \times \mathrm{YT}$, concentrated by $\mathrm{PEG} / \mathrm{NaCl}$ precipitation and resuspended in $1 \mathrm{ml} 10 \mathrm{mM}$ Tris- $\mathrm{HCl}$ (pH 7.6).

To eliminate structurally unstable virions (lacking pIII; derived from non-secretome library clones), extracted phagemid particles were incubated in $0.1 \%(\mathrm{w} / \mathrm{v})$ sarcosyl for $10 \mathrm{~min}$ at room temperature. The ssDNA released from defective virions was removed by incubation with DNaseI $(200 \mathrm{U})$ in the presence of $\mathrm{MgCl}_{2}(5 \mathrm{mM})$ for $1 \mathrm{~h}$ at room temperature, followed by addition of EDTA (to final concentration of $25 \mathrm{mM}$ ) and heating at $75^{\circ} \mathrm{C}$ for $10 \mathrm{~min}$ to inactivate DNase. Sarcosyl-resistant recombinant virions were precipitated by PEG/ $\mathrm{NaCl}$ and the ssDNA was extracted using E.Z.N.A. M13 DNA Kit (Omega Bio-Tek, USA) according to manufacturer's recommendations.

\section{Construction of pilot metasecretome library and sequence analysis of randomly selected metasecretome library inserts}

The ssDNA isolated after the secretome selection was transformed into E. coli and inserts from individual transformants analysed by Sanger sequencing. In the pilot experiment, DNA from 90 randomly selected transformants were sequenced at the Massey Genome Service (Massey University, New Zealand). All inserts were sequenced using primer pspR03 (5' -TGCCTTTAGCGTCAGACTG TAGC-3'), complementary to the pIII-coding sequence of the vector to identify the insert-pIII joint and determine the frame of the insert-containing ORF relative to pIII. The sequences obtained were analysed using Vector $\mathrm{NTI}^{\odot}$ Advance 11 Software package (Life Technologies, USA). Types of secretion signals in putative ORFs (longer than 24 amino acid residues) in frame with phage gIII were predicted using a range of available algorithms (SignalP 4.1 [33], TMHMM 2.0 [35], LipoP 1.0 [70], PRED-LIPO [36], SecretomeP 2.0 [71], PilFind 1.0 [72], PRED-TAT [73]) using the default settings and cut-off values.

\section{Next generation sequencing sample preparation}

The secretome-selected ssDNA derived from the largescale primary library through 27 separate ligations, library amplifications and selections was amplified in 27 separate PCR reactions (35 cycles starting from picogram amounts of ssDNA template) using hot-start PrimeSTAR ${ }^{\circ}$ Max DNA Polymerase (Takara Bio, Japan). Primers PCRF2 (5' -GCC TGGTATCTTTATAGTCCTGTCGGGTTTCGCCA-3') and PCRR2 (5'-GGCGACATTCAACGATTGAGGGAG GGAAGGT-3') were designed to anneal to pDJ01, 361 bp upstream, and 367 bp downstream, of the library insert. Analysis of each of the 27 PCR reactions by agarose gel electrophoresis showed smears of different-sized products, and in addition several discernable bands, suggesting more prominent amplification of some clones. The band patterns were different in all 27 PCR reactions, suggesting that there was no single highly prominent amplification product. Moreover, the Sanger sequencing reactions of the two eluted bands showed multiple traces in the chromatogram, representing a mixture of products rather than a single product. The analysis of the PCR reactions by agarose gel electrophoresis also demonstrated that the amplicon corresponding to the empty vector (728 nt) could not be detected as a separate band. Empty vector was the single most abundant clone in the 
metagenomic library prior to selection, and the lack of its amplification using post-selection DNA as a template confirmed that the secretome selection step eliminated most of the "background" non-secretome-encoding recombinant phagemids, including the empty vector.

Amplicons generated in these 27 PCR reactions were pooled and fragmented by two shearing methods: restriction endonuclease AluI (Thermo Fisher Scientific, USA) treatment and mechanical shearing using nebulisers, under several conditions (see below), to obtain a fragment length range between 0.6 and $0.8 \mathrm{~kb}$ recommended for pyrosequencing. The sample was divided into portions and fragmented using five different conditions: $1 \mathrm{~min}$ AluI digestion; $3 \mathrm{~h}$ AluI digestion, 6 min nebulisation at 35 psi; 6 min nebulisation at 35 psi followed by $1 \mathrm{~min}$ AluI digestion, and 6 min nebulisation followed by $3 \mathrm{~h}$ AluI digestion. AluI digestions were performed with $5 \mathrm{U}$ enzyme/ $\mu \mathrm{g}$ DNA at $37^{\circ} \mathrm{C}$ and to stop the enzymatic reactions, AluI was inactivated by heating at $65^{\circ} \mathrm{C}$ for 20 min. Mechanical shearing of samples containing 10\% $(\mathrm{v} / \mathrm{v})$ of glycerol was performed on ice, in a disposable nebuliser (Invitrogen, USA), by applying pressure at $35 \mathrm{psi}$ for $6 \mathrm{~min}$. Equal amounts $(2.5 \mu \mathrm{g})$ of DNA, sizefractionated by all five methods, were mixed and a total of $12.5 \mu \mathrm{g}$ DNA was submitted to pyrosequencing using 454. GS FLX Titanium platform (Roche, Switzerland) at Macrogen Inc. sequencing facility (Seoul, Korea; a halfplate in total). Sequencing template was prepared by the sequencing-service provider according to the Rapid Library Preparation Method Manual (Roche, Switzerland), except that the protocol commenced from the second, fragment end repair step.

\section{In silico analysis of NGS metasecretome dataset}

Metasecretome pyrosequencing reads were trimmed with SeqClean [74] to remove sequences of $\mathrm{pDJ} 01$ vector and VCSM13d3 helper phage. Summary statistics for metasecretome reads are presented in Additional file 2. Metagenome sequence dataset obtained by shotgun sequencing of the total metagenomic DNA from the plantadherent rumen microbial communities of two New Zealand cows, grazing a similar pasture-based diet to the cow used for the metasecretome library analysis, using Roche 454 GS FLX platform (one plate per cow; two plates in total) was analysed to provide a reference point for comparison to the metasecretome dataset. Both sequencing datasets were processed and automatically annotated using the JGI IMG/M system [50]. Functional categorisation and phylogenetic composition of annotated metasecretome and metagenome sequence datasets can be accessed through IMG/M system [75].

Protein coding genes predicted via the IMG/M system for the metasecretome and metagenome datasets $(222,960$ and 671,876 ORFs, respectively), as well as
2,547,270 predicted ORFs from the bovine switchgrassadherent metagenome dataset [14], were subjected to annotation and assignment to families of carbohydrateactive enzymes (CAZymes) using dbCAN database release 3.0, based on the CAZy database as of March 2013 [54]. dbCAN output was parsed using the following cutoff values: alignment length $>80$ amino acid residues, Evalue $<1 \times 10^{-5}$; otherwise E-value $<1 \times 10^{-3}$. To remove duplicates and to analyse distinct ORFs, all dbCAN hits were clustered at $100 \%$ sequence identity threshold using CD-HIT algorithm [76] and clustered hits to cellulosomeassociated modules were further analysed. The family level taxonomic assignment of ORFs containing cellulosome modules in the metasecretome was analysed based on the best BLASTP hit against the NCBI-NR database. For hits with a 40 bit-score threshold for cohesin and SLH module-containing ORFs, and a 35 bit-score threshold for dockerin-module containing ORFs, taxonomic family assignments of the host organism for the best BLAST hit were manually curated using recent bacterial classification proposals [56,77-81].

\section{Availability of supporting data}

The pilot metasecretome phage display library sequences supporting the results of this article are available in the GenBank repository and their accession numbers are included within Additional file 1. The metasecretome and metagenome sequence datasets supporting the results of this article can be accessed through the 'quick genome search' box available on the IMG/M main page using the corresponding IMG genome ID (3300000332 for metasecretome and 3300000524 for metagenome dataset), or in the NCBI BioProject database (accession ID PRJNA244109).

\section{Additional files}

Additional file 1: Predicted membrane targeting signals and annotation of putative ORFs in the metasecretome pilot library.

Additional file 2: Summary statistics of the rumen metasecretome pyrosequencing dataset.

Additional file 3: Carbohydrate-active enzymes and associated modules identified in the rumen plant-adherent microbial metasecretome.

Additional file 4: Whole rumen content fractionation.

\section{Abbreviations}

$2 \times$ YT: $2 \times$ Yeast extract Tryptone; Cm: Chloramphenicol; $2 \times$ YTCm $_{25}: 2 \times$ YT broth or agar supplemented with $25 \mathrm{\mu g} \mathrm{ml}^{-1}$ chloramphenicol; soft agar: $2 \times$ YT broth containing $0.6 \%(\mathrm{w} / \mathrm{v})$ molecular biology grade agarose; Double-layer selective plates: $2 \times$ YT $\mathrm{Cm}_{25}$ plates overlaid with $\mathrm{Cm}$-free $2 \times$ YT agar shortly before use; PEG: Polyethylene glycol; ORF: Open reading frame; sSDNA: Single-stranded DNA; NGS: Next-generation sequencing; HMM: Hidden Markov Model. 


\section{Competing interests}

The authors declare that there are no financial or non-financial competing interests in the publication of this manuscript. Author $M C$ is a postgraduate student of Massey University, New Zealand who has conducted her thesis research at AgResearch Ltd under the supervision of DG, CDM, SCL, EA and GTA, who are are all employees of AgResearch Ltd and JR who is employee of Massey University.

\section{Authors' contributions}

MC carried out experimental work and bioinformatic analyses. The metasecretome selection method was designed by DG and JR and optimized by MC. Bioinformatic analyses were carried out by MC, CC, SL, EA, $D G$ and $C M$. The manuscript was written by DG and MC. DG, JR, CM, GA had advisory roles in the aspects of library construction and together with SL, CC and EA in bioinformatic analyses. All co-authors had input into reviewing the manuscript. All authors read and approved the final manuscript.

\section{Acknowledgments}

This work was funded by the New Zealand Ministry of Business, Innovation and Employment (contract C10X0803). MC was partially supported by the Institute of Fundamental Sciences (Massey University). We are grateful to Roger Moraga Martinez (AgResearch) for advice on bioinformatic analysis of metasecretome sequences, Dr Garry Waghorn (DairyNZ) for providing rumen samples, Carrie Sang (AgResearch) for help with rumen content fractionation, Dr Bill Kelly (AgResearch) and Dr Ron Ronimus (AgResearch) for useful suggestions regarding annotation of CAZymes and Dr Yanbin Yin (Northern Illinois University, IL, USA) for help with using dbCAN database.

\section{Author details}

${ }^{1}$ Animal Nutrition and Health, AgResearch Ltd, Palmerston North 4442, New Zealand. ${ }^{2}$ Institute of Fundamental Sciences, Massey University, Palmerston North 4442, New Zealand. ${ }^{3}$ Institute of Biological, Environmental \& Rural Sciences, Aberystwyth University, Penglais, Aberystwyth, Ceredigion SY23 3DA, UK.

Received: 5 November 2013 Accepted: 29 April 2014 Published: 12 May 2014

\section{References}

1. Cowan DA: Microbial genomes - the untapped resource. Trends Biotechnol 2000, 18(1):14-16.

2. Cowan D, Meyer Q, Stafford W, Muyanga S, Cameron R, Wittwer P. Metagenomic gene discovery: past, present and future. Trends Biotechnol 2005, 23(6):321-329.

3. Amann Rl, Ludwig W, Schleifer KH: Phylogenetic identification and in situ detection of individual microbial cells without cultivation. Microbiol Rev 1995, 59(1):143-169.

4. Handelsman J: Metagenomics: application of genomics to uncultured microorganisms. Microbiol Mol Biol Rev 2004, 68(4):669-685.

5. Streit WR, Daniel R, Jaeger KE: Prospecting for biocatalysts and drugs in the genomes of non-cultured microorganisms. Curr Opin Biotechnol 2004, 15(4):285-290.

6. Xing MN, Zhang $X Z$, Huang $H$ : Application of metagenomic techniques in mining enzymes from microbial communities for biofuel synthesis. Biotech Adv 2012, 30(4):920-929.

7. Ferrer M, Golyshina OV, Chernikova TN, Khachane AN, Reyes-Duarte D, Santos VA, Strompl C, Elborough K, Jarvis G, Neef A, Yakimov MM, Timmis KN, Golyshin PN: Novel hydrolase diversity retrieved from a metagenome library of bovine rumen microflora. Environ Microbiol 2005, 7(12):1996-2010.

8. Morgavi DP, Kelly WJ, Janssen PH, Attwood GT: Rumen microbial (meta) genomics and its application to ruminant production. Animal 2012, 7(s1):184-201.

9. Williams AG, Withers SE: Hemicellulose-degrading enzymes synthesized by rumen bacteria. J App/ Bacterio/ 1981, 51(2):375-385.

10. Cotta MA: Amylolytic activity of selected species of ruminal bacteria. Appl Environ Microbiol 1988, 54(3):772-776.

11. Whitehead TR, Hespell RB: Cloning and expression in Escherichia coli of a xylanase gene from Bacteroides ruminicola 23. Appl Environ Microbiol 1989, 55(4):893-896.
12. Fouts DE, Szpakowski S, Purushe J, Torralba M, Waterman RC, MacNeil MD, Alexander $\amalg$, Nelson KE: Next generation sequencing to define prokaryotic and fungal diversity in the bovine rumen. PLoS One 2012, 7(11):e48289.

13. Brulc JM, Antonopoulos DA, Miller ME, Wilson MK, Yannarell AC, Dinsdale EA, Edwards RE, Frank ED, Emerson JB, Wacklin P, Coutinho PM, Henrissat B, Nelson KE, White BA: Gene-centric metagenomics of the fiber-adherent bovine rumen microbiome reveals forage specific glycoside hydrolases. Proc Natl Acad Sci USA 2009, 106(6):1948-1953.

14. Hess M, Sczyrba A, Egan R, Kim TW, Chokhawala H, Schroth G, Luo S, Clark DS, Chen F, Zhang T, Mackie RI, Pennacchio LA, Tringe SG, Visel A, Woyke T, Wang Z, Rubin EM: Metagenomic discovery of biomass-degrading genes and genomes from cow rumen. Science 2011, 331(6016):463-467.

15. Kim M, Morrison M, Yu Z: Status of the phylogenetic diversity census of ruminal microbiomes. FEMS Microbiol Ecol 2011, 76(1):49-63.

16. Himmel ME, Ding SY, Johnson DK, Adney WS, Nimlos MR, Brady JW, Foust TD: Biomass recalcitrance: engineering plants and enzymes for biofuels production. Science 2007, 315(5813):804-807.

17. Cantarel BL, Coutinho PM, Rancurel C, Bernard T, Lombard V, Henrissat B: The Carbohydrate-Active EnZymes database (CAZy): an expert resource for Glycogenomics. Nucleic Acids Res 2009, 37(Database issue):D233-D238.

18. Cuskin F, Flint JE, Gloster TM, Morland C, Basle A, Henrissat B, Coutinho PM Strazzulli A, Solovyova AS, Davies GJ, Gilbert HJ: How nature can exploit nonspecific catalytic and carbohydrate binding modules to create enzymatic specificity. Proc Natl Acad Sci U S A 2012, 109(51):20889-20894.

19. Blake AW, McCartney L, Flint JE, Bolam DN, Boraston AB, Gilbert HJ, Knox JP: Understanding the biological rationale for the diversity of cellulosedirected carbohydrate-binding modules in prokaryotic enzymes. J Biol Chem 2006, 281(39):29321-29329.

20. Boraston AB, Bolam DN, Gilbert HJ, Davies GJ: Carbohydrate-binding modules: fine-tuning polysaccharide recognition. Biochem J 2004, 382(Pt 3):769-781.

21. Doi RH, Kosugi A: Cellulosomes: plant-cell-wall-degrading enzyme complexes. Nat Rev Microbiol 2004, 2(7):541-551.

22. Bayer EA, Lamed R, White BA, Flints HJ: From cellulosomes to cellulosomics. Chem Rec 2008, 8(6):364-377.

23. Bayer EA, Setter E, Lamed R: Organization and distribution of the cellulosome in Clostridium thermocellum. J Bacteriol 1985, 163(2):552-559.

24. Lamed R, Setter E, Bayer EA: Characterization of a cellulose-binding, cellulase-containing complex in Clostridium thermocellum. J Bacteriol 1983, 156(2):828-836.

25. Fierobe HP, Bayer EA, Tardif C, Czjzek M, Mechaly A, Belaich A, Lamed R, Shoham Y, Belaich JP: Degradation of cellulose substrates by cellulosome chimeras. Substrate targeting versus proximity of enzyme components. J Biol Chem 2002, 277(51):49621-49630.

26. Tasse L, Bercovici J, Pizzut-Serin S, Robe P, Tap J, Klopp C, Cantarel BL, Coutinho PM, Henrissat B, Leclerc M, Dore J, Monsan P, Remaud-Simeon M, Potocki-Veronese G: Functional metagenomics to mine the human gut microbiome for dietary fiber catabolic enzymes. Genome Res 2010, 20(11):1605-1612.

27. Maione D, Margarit I, Rinaudo CD, Masignani V, Mora M, Scarselli M, Tettelin $H$, Brettoni C, lacobini ET, Rosini R, D'Agostino N, Mirion L, Buccato S, Mariani M, Galli G, Nogarotto R, Dei VN, Vegni F, Fraser C, Mancuso G, Teti G, Madoff LC, Paoletti LC, Rappuoli R, Kasper DL, Telford JL, Grandi G: Immunology: Identification of a universal group B Streptococcus vaccine by multiple genome screen. Science 2005, 309(5731):148-150.

28. Boekhorst J, Wels M, Kleeberezem M, Siezen RJ: The predicted secretome of Lactobacillus plantarum WCFS1 sheds light on interactions with its environment. Microbiology 2006, 152(11):3175-3183.

29. Hammerschmidt S: Adherence molecules of pathogenic pneumococci. Curr Opin Biotechnol 2006, 9(1):12-20.

30. Leary DH, Hervey WJ, Deschamps JR, Kusterbeck AW, Vora GJ: Which metaproteome? The impact of protein extraction bias on metaproteomic analyses. Mol Cell Probe 2013, 27:193-199.

31. Erickson AR, Cantarel BL, Lamendella R, Darzi $Y$, Mongodin EF, Pan C, Shah M, Halfvarson J, Tysk C, Henrissat B: Integrated metagenomics/ metaproteomics reveals human host-microbiota signatures of Crohn's disease. PloS One 2012, 7(11):e49138.

32. Economou A: Bacterial secretome: the assembly manual and operating instructions (review). Molec Membr Biol 2002, 19(3):159-169. 
33. Petersen TN, Brunak S, Von Heijne G, Nielsen H: SignalP 4.0: discriminating signal peptides from transmembrane regions. Nat Methods 2011, 8(10):785-786

34. Bendtsen JD, Jensen LJ, Blom N, Von Heijne G, Brunak S: Feature-based prediction of non-classical and leaderless protein secretion. Protein Eng Des Sel 2004, 17(4):349-356.

35. Krogh A, Larsson B, Von Heijne G, Sonnhammer EL: Predicting transmembrane protein topology with a hidden Markov model: application to complete genomes. J Mol Biol 2001, 305(3):567-580.

36. Bagos PG, Tsirigos KD, Liakopoulos TD, Hamodrakas SJ: Prediction of lipoprotein signal peptides in Gram-positive bacteria with a Hidden Markov Model. J Proteome Res 2008, 7(12):5082-5093.

37. Rusch DB, Halpern AL, Sutton G, Heidelberg KB, Williamson S, Yooseph $\mathrm{S}$, Wu D, Eisen JA, Hoffman JM, Remington K, Beeson K, Tran B, Smith $H$, Baden-Tillson H, Stewart C, Thorpe J, Freeman J, Andrews-Pfannkoch C, Venter JE, Li K, Kravitz S, Heidelberg JF, Utterback T, Rogers YH, Falcon LI, Souza V, Bonilla-Rosso G, Eguiarte LE, Karl DM, Sathyendranath S, et al: The Sorcerer II Global Ocean Sampling expedition: northwest Atlantic through eastern tropical Pacific. PLoS Biol 2007, 5(3):e77.

38. Gill SR, Pop M, DeBoy RT, Eckburg PB, Turnbaugh PJ, Samuel BS, Gordon J, Relman DA, Fraser-Liggett CM, Nelson KE: Metagenomic analysis of the human distal gut microbiome. Science 2006, 312(5778):1355-1359.

39. Prakash T, Taylor TD: Functional assignment of metagenomic data: challenges and applications. Brief Bioinform 2012, 13(6):711-727.

40. Jankovic D, Collett MA, Lubbers MW, Rakonjac J: Direct selection and phage display of a Gram-positive secretome. Genome Biol 2007, 8(12):R266.

41. Liu S, Han W, Sun C, Lei L, Feng X, Yan S, Diao Y, Gao Y, Zhao H, Liu Q, Yao C, Li M: Subtractive screening with the Mycobacterium tuberculosis surface protein phage display library. Tuberculosis (Edinb) 2011, 91(6):579-586

42. Gagic D, Wen W, Collett MA, Rakonjac J: Unique secreted-surface protein complex of Lactobacillus rhamnosus, identified by phage display. Microbiol Open 2012, 2:1-17.

43. Liu SS, Han WY, Sun CJ, Lei LC, Feng X, Zu S, Zai ZD, Gao Y, Zhao HL, Yao CM: Identification of two new virulence factors of Mycobacterium tuberculosis that induce multifunctional CD4 T cell responses. J Mycobac Dis 2013, 3(1):S6.

44. Rakonjac J, Bennett NJ, Spagnuolo J, Gagic D, Russel M: Filamentous bacteriophage: biology, phage display and nanotechnology applications. Curr Issues Mol Biol 2011, 13(2):51-76.

45. Zwick MB, Shen J, Scott JK: Phage-displayed peptide libraries. Curr Opin Biotechnol 1998, 9(4):427-436.

46. Barbas CF III, Burton DR, Scott JK, Silverman GJ: Phage Display: A Laboratory Manual. Cold Spring Harbor, New York: Cold Spring Harbor Laboratory Press; 2001.

47. Paschke $M$, Höhne W: A twin-arginine translocation (Tat)-mediated phage display system. Gene 2005, 350(1):79-88.

48. Paschke M: Phage display systems and their applications. App/ Microbio/ Biotechnol 2006, 70(1):2-11.

49. Jami E, Mizrahi I: Composition and similarity of bovine rumen microbiota across individual animals. PLoS One 2012, 7(3):e33306.

50. Markowitz VM, Chen IM, Chu K, Szeto E, Palaniappan K, Grechkin Y, Ratner A, Jacob B, Pati A, Huntemann M, Liolios K, Pagani I, Anderson I, Mavromatis K, Ivanova NN, Kyrpides NC: IMG/M: the integrated metagenome data management and comparative analysis system. Nucleic Acids Res 2012, 40(Database issue):D123-D129.

51. Punta M, Coggill PC, Eberhardt RY, Mistry J, Tate J, Boursnell C, Pang N, Forslund K, Ceric G, Clements J, Heger A, Holm L, Sonnhammer EL, Eddy SR, Bateman A, Finn RD: The Pfam protein families database. Nucleic Acids Res 2012, 40(Database issue):D290-D301.

52. Antelmann H, Tjalsma H, Voigt B, Ohlmeier S, Bron S, Dijl J, Hecker M: A proteomic view on genome-based signal peptide predictions. Genome Res 2001, 11:1484-1502.

53. Lichanska AM: Secreted bacterial proteins. Genome Biol 2001 2(12):reports0047.

54. Yin $Y$, Mao $X$, Yang J, Chen $X$, Mao $F, X u$ Y: dbCAN: a web resource for automated carbohydrate-active enzyme annotation. Nucleic Acids Res 2012, 40(Web Server issue):W445-W451.

55. Dell A, Galadari A, Sastre F, Hitchen P: Similarities and differences in the glycosylation mechanisms in prokaryotes and eukaryotes. Int J Microbiol 2010, 2010:148-178.
56. Yutin N, Galperin MY: A genomic update on clostridial phylogeny: gramnegative spore formers and other misplaced clostridia. Environ Microbiol 2013, 10(15):2631-2641.

57. Whitman WB, Goodfellow M, Kämpfer P, Busse H-J, Trujillo ME, Ludwig W, Suzuki K-i, Parte A: Bergey's Manual ${ }^{\oplus}$ of Systematic Bacteriology, Volume 5. 2nd edition. New York: Springer; 2012

58. Woodward R, Yi W, Li L, Zhao G, Eguchi H, Sridhar PR, Guo H, Song JK, Motari E, Cai L, Kelleher P, Liu X, Han W, Zhang W, Ding Y, Li M, Wang PG: In vitro bacterial polysaccharide biosynthesis: defining the functions of Wzy and Wzz. Nat Chem Biol 2010, 6(6):418-423.

59. Jindou S, Borovok I, Rincon MT, Flint HJ, Antonopoulos DA, Berg ME, White BA, Bayer EA, Lamed R: Conservation and divergence in cellulosome architecture between two strains of Ruminococcus flavefaciens. J Bacteriol 2006, 188(22):7971-7976.

60. Rincon MT, Ding SY, McCrae SI, Martin JC, Aurilia V, Lamed R, Shoham Y, Bayer EA, Flint HJ: Novel organization and divergent dockerin specificities in the cellulosome system of Ruminococcus flavefaciens. J Bacteriol 2003, 185(3):703-713.

61. Dai X, Zhu Y, Luo Y, Song L, Liu D, Liu L, Chen F, Wang M, Li J, Zeng X Dong Z, Hu S, Li L, Xu J, Huang L, Dong X: Metagenomic insights into the fibrolytic microbiome in yak rumen. PloS ONE 2012, 7(7):e40430.

62. Pope PB, Mackenzie AK, Gregor I, Smith W, Sundset MA, McHardy AC, Morrison M, Eijsink VG: Metagenomics of the Svalbard reindeer rumen microbiome reveals abundance of polysaccharide utilization loci. PLOS One 2012, 7(6):e38571.

63. Lamed R, Morag E, Mor-Yosef O, Bayer E: Cellulosome-like entities in Bacteroides cellulosolvens. Curr Microbiol 1991, 22:27-33.

64. Peer A, Smith SP, Bayer EA, Lamed R, Borovok I: Noncellulosomal cohesinand dockerin-like modules in the three domains of life. FEMS Microbiol Lett 2009, 291(1):1-16.

65. Forrer $P$, Jung $S$, Plückthun $A$ : Beyond binding: using phage display to select for structure, folding and enzymatic activity in proteins. Curr Opin Struc Biol 1999, 9(4):514-520.

66. Demartis S, Huber A, Viti F, Lozzi L, Giovannoni L, Neri P, Winter G, Neri D: A strategy for the isolation of catalytic activities from repertoires of enzymes displayed on phage. J Mol Biol 1999, 286(2):617-633.

67. Rakonjac J, Jovanovic G, Model P: Filamentous phage infection-mediated gene expression: construction and propagation of the glll deletion mutant helper phage R408d3. Gene 1997, 198(1-2):99-103.

68. Stein J, Marsh T, Wu K, Shizuya H, DeLong E: Characterization of uncultivated prokaryotes: isolation and analysis of a 40-kilobase-pair genome fragment from a planktonic marine archaeon. J Bacteriol 1996, 178(3):591.

69. Russel M: Protein-protein interactions during filamentous phage assembly. J Mol Biol 1993, 231(3):689-697.

70. Juncker AS, Willenbrock H, Von Heijne G, Brunak S, Nielsen H, Krogh A: Prediction of lipoprotein signal peptides in Gram-negative bacteria. Protein Sci 2003, 12(8):1652-1662.

71. Bendtsen JD, Kiemer L, Fausbø\|l A, Brunak S: Non-classical protein secretion in bacteria. BMC Microbio/ 2005, 5(1):58.

72. Imam S, Chen Z, Roos DS, Pohlschröder M: Identification of surprisingly diverse type IV pili, across a broad range of Gram-positive bacteria. PLoS One 2011, 6(12):e28919.

73. Bagos PG, Nikolaou EP, Liakopoulos TD, Tsirigos KD: Combined prediction of Tat and Sec signal peptides with hidden Markov models. Bioinformatics 2010, 26(22):2811-2817.

74. SeqClean. [http://seqclean.sourceforge.net/].

75. The Integrated Microbial Genomes and Metagenomes (IMG/M) system. [https://img.jgi.doe.gov/cgi-bin/m/main.cgi].

76. Huang Y, Niu B, Gao Y, Fu L, Li W: CD-HIT Suite: a web server for clustering and comparing biological sequences. Bioinformatics 2010, 26(5):680-682.

77. Carlier JP, Bedora-Faure M, K'Ouas G, Alauzet C, Mory F: Proposal to unify Clostridium orbiscindens Winter et al. 1991 and Eubacterium plautii (Seguin 1928) Hofstad and Aasjord 1982, with description of Flavonifractor plautii gen. nov., comb. nov., and reassignment of Bacteroides capillosus to Pseudoflavonifractor capillosus gen. nov., comb. nov. Int J Syst Evol Microbiol 2010, 60(Pt 3):585-590.

78. Downes J, Dewhirst FE, Tanner AC, Wade WG: Description of Alloprevotella rava gen. nov., sp. nov., isolated from the human oral cavity, and reclassification of Prevotella tannerae Moore et al. 1994 as Alloprevotella 
tannerae gen. nov., comb. nov. Int J Syst Evol Microbiol 2013, 63(Pt 4):1214-1218.

79. Quast C, Pruesse E, Yilmaz P, Gerken J, Schweer T, Yarza P, Peplies J, Glockner FO: The SILVA ribosomal RNA gene database project: improved data processing and web-based tools. Nucleic Acids Res 2013, 41(Database issue):D590-D596.

80. Krieg N, Ludwig W, Euzéby J, Whitman W: Phylum XIV. Bacteroidetes phyl. nov. In Bergey's Manual ${ }^{\circledR}$ of Systematic Bacteriology. Edited by Krieg N, Staley J, Brown D, Hedlund B, Paster B, Ward N, Ludwig W, Whitman W. New York: Springer; 2010:25-469.

81. Schleifer K-H: Phylum XIII.Firmicutes Gibbons and Murray 1978, 5. In Bergey's Manual ${ }^{\oplus}$ of Systematic Bacteriology. Edited by Vos P, Garrity G, Jones D, Krieg N, Ludwig W, Rainey F, Schleifer K-H, Whitman W. New York: Springer: 2009:19-1317.

doi:10.1186/1471-2164-15-356

Cite this article as: Ciric et al:: Metasecretome-selective phage display approach for mining the functional potential of a rumen microbial community. BMC Genomics 2014 15:356.

\section{Submit your next manuscript to BioMed Central and take full advantage of:}

- Convenient online submission

- Thorough peer review

- No space constraints or color figure charges

- Immediate publication on acceptance

- Inclusion in PubMed, CAS, Scopus and Google Scholar

- Research which is freely available for redistribution 